\title{
Causal exclusion without causal sufficiency
}

\author{
Bram Vaassen ${ }^{1}$ \\ Received: 19 August 2019 / Accepted: 27 May 2020 / Published online: 15 June 2020 \\ (c) The Author(s) 2020
}

\begin{abstract}
Some non-reductionists claim that so-called 'exclusion arguments' against their position rely on a notion of causal sufficiency that is particularly problematic. I argue that such concerns about the role of causal sufficiency in exclusion arguments are relatively superficial since exclusionists can address them by reformulating exclusion arguments in terms of physical sufficiency. The resulting exclusion arguments still face familiar problems, but these are not related to the choice between causal sufficiency and physical sufficiency. The upshot is that objections to the notion of causal sufficiency can be answered in a straightforward fashion and that such objections therefore do not pose a serious threat to exclusion arguments.
\end{abstract}

Keywords Exclusion argument · Causal exclusion · Causal sufficiency · Non-reductionism · Causation · Philosophy of mind · Mental causation · Reductionism

\section{Introduction}

Some philosophers maintain that so-called exclusion arguments support the conclusion that only physical phenomena can cause physical effects. These philosophers argue that non-reductionist theories of the mind therefore cannot allow mental phenomena to be causes of physical effects (Kim 1989, 2005; Ney 2012; Papineau 2002). ${ }^{1}$ Nonreductionists often claim that such exclusion arguments rely on a problematic notion of causal sufficiency. I will argue that these concerns about the role of causal sufficiency in exclusion arguments are relatively superficial. One can formulate valid exclusion arguments that support the same conclusion but do not rely on the notion of causal sufficiency. Consequently, such objections do not pose a serious threat to exclusion arguments.

\footnotetext{
1 I use 'phenomena' as a place-holder for the reader's preferred causal relata. Nothing turns on this.

$凶$ Bram Vaassen

bram.vaassen@umu.se

1 Department of Philosophy, University of Cologne, Cologne, Germany
} 
In Sect. 2, I provide a standard formulation of an exclusion argument and discuss some of the objections to its reliance on the notion of causal sufficiency. In Sect. 3, I introduce the notion of physical sufficiency and reformulate the two premises of the standard argument that rely on the notion of causal sufficiency in terms of physical sufficiency. I then show how these reformulated premises avoid the kinds of objections discussed in Sect. 2. In Sect. 4, I conclude that the resulting argument is valid and that remaining concerns about its soundness are not related to the choice between causal sufficiency and physical sufficiency. The upshot is that the objections to the role of causal sufficiency in exclusion arguments can be answered in a straightforward fashion and that such objections therefore do not pose a serious threat to exclusion arguments.

\section{Non-reductionists vs. causal sufficiency}

Non-reductionists about the mind share one commitment. Namely that mental phenomena are distinct from physical phenomena in the following sense: no mental phenomenon is identical to a physical phenomenon. ${ }^{2}$ Causal exclusion arguments against such non-reductionism have many formulations. We can take the following formulation, loosely based on Bennett (2008), as a starting point:

Mental Causation Mental phenomena systematically cause physical phenomena.

Non-Reductionism Mental phenomena are distinct from physical phenomena.

Causal Closure Every physical phenomenon has a sufficient physical cause at any given time $t$ (if it has a cause at all at $t$ ).

Physical Parts For any two phenomena $A$ and $B$, if $A$ is a physical phenomenon and $B$ is a part of $A$, then $B$ is a physical phenomenon as well. ${ }^{3}$

Causal Exclusion For any three phenomena $A, B$ and $C$ : if $A$ occurs at $t$ and is a sufficient cause for $B$ 's occurrence at $t+x$, no phenomenon $C$ occurring at $t$ that is distinct from $A$ and is distinct from all of $A$ 's parts is a cause of $B$, unless it is a case of genuine overdetermination. ${ }^{4}$

Non-Overdetermination There is no systematic genuine overdetermination of physical effects with mental causes.

Mental Causation and Non-Reductionism together entail that physical phenomena are systematically caused by phenomena that are distinct from physical phenomena.

\footnotetext{
${ }^{2}$ Unless specified otherwise, I will take 'distinct' to mean non-identical in what follows. Note that some, like Kim (2005) are non-reductionists about some mental phenomena, but not about others. I ignore this complication here, as one can reformulate the exclusion arguments to affect only those mental phenomena that are distinct from physical phenomena.

${ }^{3}$ By 'part of' I mean something along the lines of 'mereological part of'. Given that mereological parthood is liberal about what it can take as relata (cf. Johnston 2006, pp. 654-655), this reading allows us to remain non-committal about what the place-holder term 'phenomena' should refer to (cf. footnote 1). I am indebted to an anonymous referee for a comment that prompted me to include this premise.

${ }^{4}$ For the sake of simplicity, I assume throughout the rest of the text that, for any phenomenon $X$, being distinct from $X$ requires being distinct from all of $X$ 's parts. I will use reminders like 'and is distinct from all of $X$ 's parts' in definitions.
} 
Causal Closure, Physical Parts and Causal Exclusion together entail that there are no phenomena that are distinct from physical phenomena and cause physical phenomena, unless it is a case of genuine overdetermination. Finally, Non-Overdetermination states that systematic genuine overdetermination is not an option. One of these six has to go. The exclusionist concludes that Non-Reductionism has to go: we should deny that mental phenomena are distinct from physical phenomena.

When responding, non-reductionists frequently remark that exclusion arguments rely on the notion of causal sufficiency and claim that this notion is problematic. For example, Menzies claims that:

The fundamental error of this [causal exclusion] principle is that it mistakes causal sufficiency for causation (Menzies 2013, p. 71).

Raatikainen makes a similar claim:

[...] both these assumptions [i.e. Causal Closure and Causal Exclusion] involve confusing causes with sufficient conditions. There are causes, which are difference-makers; and there are sufficient conditions, which are wholly different issues and not causes of any sort; there are no such things as sufficient causes. Hence, I do not think that these two assumptions are so much false (or true) as mongrels based on a conceptual confusion which fail to make clear sense (Raatikainen 2010, p. 360).

Many others make mention of the problems they find in the notion of causal sufficiency and its role in the exclusion argument. Examples can be found in Crane and Arnadottir (2013), Hitchcock (2012), Koons and Bealer (2010), List and Menzies (2009), Menzies (2015), Pernu (2013, 2016), Raatikainen (2013, 2018), Woodward (2008) and Zhong (2019). Such objections to the reliance on causal sufficiency tend to be part of more integrated responses to exclusion arguments. I do not intend to question the ultimate viability of these responses here. Instead, I will argue that, whatever their success, it cannot be due to such objections to causal sufficiency, because exclusion arguments can be formulated without relying on that notion. This indicates that objections to the notion of causal sufficiency, rather than addressing a fundamental error in exclusion arguments, only pose a relatively superficial problem for exclusion arguments. Let us first look at what makes the notion so problematic.

A sufficient cause of an effect can intuitively be understood to be a cause of the effect that on its own suffices for the occurrence of that effect. Upon closer scrutiny, it appears that any phenomenon that is sufficient for the occurrence of an effect will have to be both enormous and maximally specific. ${ }^{5}$ This makes any phenomenon that is sufficient for an effect an unlikely candidate for being a cause of that effect.

It is easy to see why effects typically cannot have sufficient causes that we are familiar with. For example, there are many familiar causes of the window shattering after Yue threw a rock at it: Yue's throw, the rock's mass, the window's brittleness,

\footnotetext{
5 Alternatively, a sufficient cause could be understood to be sufficient for the effect given an agreed upon set of background conditions. This alternative reading will plausibly avoid the size problem, but not the specificity problem. As the aim of this section is to rehearse the non-reductionist's criticism on the notion, I focus on the reading that is most susceptible to it.
} 
etc. None of these familiar causes independently suffices for the window to shatter, as its shattering requires all of these familiar causes to occur. ${ }^{6}$ For a cause simultaneous with Yue's throw to be sufficient for the window shattering, its occurrence will have to fix the occurrence of all these familiar causes.

In fact, the occurrence of an effect requires more than just the occurrence of all its familiar causes. If these familiar causes are to give rise to the effect, there cannot be any interference with the causal process leading up to the effect. Consequently, the occurrence of a sufficient cause of an effect has to make it impossible that any such interference should occur. For example, the phenomenon simultaneous with Yue's throw that is sufficient for the window shattering has to make it impossible that there is interference by a meteor knocking the rock off course. That is a drastic requirement. Physics teaches us that an interference could travel at the speed of light. Consequently, such an interference could be on its way from an enormous distance. In order for the occurrence of a phenomenon to exclude that possibility, it has to span a sufficient amount of space, such that its occurrence fixes what is occurring at such enormous distances. Consequently, philosophers have concluded that only phenomena that span at least the entire cross-section of the backwards light cone of an effect can really be sufficient for the occurrence of that effect (e.g. Field 2003; Loewer 2007). ${ }^{7}$ In layman's terms, sufficient causes have to be enormous.

Once we start looking at what physics teaches us, it appears that sufficient causes not only have to be enormous, but also maximally specific. Any non-maximally specific phenomenon, like Yue's throw, can be physically realized such that it has a thermodynamically abnormal future. For example, the fundamental particles making up the rock might be arranged such that it suddenly emits a particle at an immense acceleration that is orthogonal to the rock's anticipated trajectory, causing the rock to make a sudden turn and therefore miss the window. Most probably, none of the actual rock throws, or enormous physical phenomena involving rock throws, will ever involve a mid-air turn due to the immensely accelerated ejection of a fundamental particle - that is why such a physical realization is thermodynamically abnormal. However, such trajectories are not physically impossible. ${ }^{8}$ In order for a phenomenon to be sufficient for an effect, its occurrence has to exclude the possibility that any of the phenomena in the relevant cross-section of the backwards light cone of that effect has a thermodynamically abnormal future that interferes with the occurrence of the effect. Not only should the rock not be poised to change its trajectory, none of the phenomena in the cross-section of the light cone can be such that they result in the ejection of a particle that will knock the rock off course. The safest way to ascertain that none of these phenomena are realized in such an abnormal way, is to fix how they actually are realized to a maximal degree of specificity. The underlying physics and theory are no doubt mind boggling, but the takeaway is quite simple: in order for any

\footnotetext{
6 Crane and Arnadottir (2013, Section 4.3) raise this point.

7 The cross-section of a backwards light cone of a phenomenon contains all space-time points at a certain time in the phenomenon's past from which a flash of light could have reached that phenomenon. If we assume that it took one second for the rock to reach the window in our example, the relevant cross-section is a sphere with a radius of approximately $300.000 \mathrm{~km}$.

8 See Albert (2015, Ch. 1) and Field (2003, p. 439) for accessible explanations.
} 
phenomenon to be sufficient for any effect, it not only has to be enormous, but also maximally specific. ${ }^{9}$

Such enormous and maximally specific phenomena would make for peculiar causes to say the least. The causes we name in everyday as well as scientific explanations, like infections and hurricanes, are significantly more local and less specific. Critics of causal sufficiency argue that it is doubtful that such enormous maximally specific phenomena even qualify to be causes according to contemporary philosophical accounts of causation, and add that accounts of causation that would treat sufficient phenomena as causes are contentious or outdated. ${ }^{10}$

This critical attitude is not unmotivated. Many philosophers take causes to be difference-makers of their effects and the absence of difference-makers ought to correlate strongly with the absence of the target effect. For example, Yue not throwing the rock correlates strongly with the window not shattering at the time it did. In contrast, no phenomenon that is enormous and specific enough to be sufficient for the window shattering can be a difference-maker for its shattering. Due to its specificity and size, any change in the relevant cross-section of the backwards light cone of the effect entails that the sufficient phenomenon does not occur. However, there are many changes in the cross-section of the backwards light cone of familiar effects that are irrelevant to these effects. For example, the physical realization of Yue's shoes or the Belgian prime minister's hairdo could have varied substantially without affecting the shattering of the window. This means that for any phenomenon that is sufficient for the window shattering, there are many scenarios in which that phenomenon did not take place, but the window nonetheless shatters at the time it did in the actual world. It therefore appears that sufficient phenomena do not correlate strongly enough with their effects to be difference-makers. If causes are difference-makers, it is unclear why any sufficient phenomenon should be considered a cause - let alone why it should outcompete mental phenomena that often do serve as difference-makers, like intentions and desires. ${ }^{11}$

If the notion of a sufficient cause does not match with our everyday, scientific or philosophical understanding of causes, it is indeed suspicious that it features in the standard formulations of two of the central premises in the exclusion argument: Causal Closure and Causal Exclusion. However, these worries can be answered in a straightforward fashion. One can formulate valid exclusion arguments that do not rely on the notion of causal sufficiency and do not encounter such worries.

\footnotetext{
9 I assume here that disjunctions of phenomena are not phenomena. Otherwise, one could construct less specific sufficient phenomena from the disjunction of maximally specific phenomena that are individually sufficient for the effect.

10 See Crane and Arnadottir (2013, p. 258), Koons and Bealer (2010, p. xix), List and Menzies (2009, p. 489), Menzies (2015, pp. 27-30) Woodward (2008, p. 251) and Raatikainen (2010) as quoted above, as well as (2013, p. 24) and (2018, p. 34). See also Hitchcock (2012, p. 53), who argues that accounts that treat sufficient phenomena as causes contradict other assumptions in exclusion arguments.

11 This is the upshot of Hitchcock (2012, pp. 53-55), List and Menzies (2009), and Menzies (2013, 2015). See also Shapiro (2010, 2012) and Shapiro and Sober (2007), who argue that the idea that mental causes can be outcompeted by the underlying physical phenomena that are (part of) a sufficient condition for their effects rests on mistaken and undermotivated assumptions about causation.
} 


\section{Physical sufficiency vs. causal sufficiency}

Getting rid of causal sufficiency is not as hard as one might expect. The following notion can do the work exclusionists cut out for causal sufficiency, without inheriting its problematic consequences:

Physical Sufficiency Phenomenon $A$ is physically sufficient for phenomenon $B$, iff all physically possible worlds which contain $A$ also contain $B .^{12}$

By 'physically possible world', I mean any possible world in which the same fundamental laws of physics as in our world hold. The rationale behind Physical Sufficiency is to make it unambiguous what is meant by saying that $A$ is 'sufficient on its own' for $B$ in the context of the exclusion argument, without making any claims about $A$ 's eligibility for being a cause of $B$ : all that is required for the occurrence of $B$ is the occurrence of $A$.

With this notion in mind, we can reformulate Causal Closure and Causal Exclusion as follows:

Physical Closure For any physical effect, and any time $t$ there is some physical phenomenon $A$ occurring at $t$ that is physically sufficient for this effect. $^{13}$

Physical Exclusion For any three phenomena $A, B$ and $C$ : if $A$ occurs at $t$ and $A$ is physically sufficient for $B$ 's occurrence at $t+x$, no phenomenon $C$ occurring at $t$ that is distinct from $A$ and is distinct from all of $A$ 's parts is a cause of $B$, unless it is a case of genuine overdetermination.

A brief look at Physical Closure and Physical Exclusion shows that they are impervious to the objections raised against the role of causal sufficiency in Causal Closure and Causal Exclusion.

Physical Closure is a standard assumption in mental causation debates. For reasons addressed earlier, physically sufficient phenomena must span vast amounts of space and must be maximally specific. Many find it plausible that such physically sufficient phenomena are never causes of familiar effects. However, Physical Closure makes no claims about $A$ being a cause and its plausibility is thus untouched by such considerations. I take it that most non-exclusionists who object to treating physically sufficient physical phenomena as causes will assent to Physical Closure. ${ }^{14}$

In fact, almost all of the authors I listed as objecting to the notion of causal sufficiency endorse Physical Closure. Some do so explicitly, like Crane and Arnadottir (2013, p. 258), Hitchcock (2012, p. 53), and Raatikainen (2010, p. 358), but all of the

\footnotetext{
$\overline{12}$ Cf. the standard definition of the $\square(p \rightarrow q)$ relation in modal logic, according to which $\square(p \rightarrow q)$ is true iff $q$ is true in all possible worlds where $p$ is true.

13 For ease of formulation, this principle assumes the truth of determinism. If required, $A$ can be said to be physically sufficient for the probability of the effect occurring rather than the occurrence of the effect. The principle also entails that physical effects have physically sufficient phenomena in their future. Although this is not essential to our argument and it is somewhat counterintuitive, it is a relatively non-controversial feature of physical necessitation that it also works in the future-to-past direction (cf. Field 2003).

14 See Lowe (2003) and Won (2019) for exceptions.
} 
listed authors, except Koons and Bealer (2010), endorse a non-reductionist physicalism that is committed to Physical Closure. And even though Koons and Bealer do not explicitly endorse Physical Closure, they do propose that the popularity of exclusion arguments is due to a confusion between Causal Closure and Physical Closure. They state that

Anti-materialism [i.e. Non-Reductionism] is alleged to be unable to accommodate the possibility of mental causation without violating the causal closure of the physical. But this is not at all clear when causal closure is formulated in its most plausible form, as follows: for every physical event $B$ that has a cause, there is a physical event $c$ such that it is nomologically (or causally) necessary that if $c$ occurs, $B$ occurs. Suppose that physics requires, and provides justification for, this weak causal closure principle. But obviously this weak principle does not imply the following stronger closure principle: for every physical event $B$ that has a cause, there is a physical event $B$ such that $c$ is a sufficient cause of $B$. Failure to appreciate the distinction between weak causal closure and strong closure principle has led many philosophers to the conclusion that mental causation is untenable in an anti-materialist setting, [...] (2010, p. xix, emphasis in the original)

Koons and Bealer suggest that the physical phenomena that are causally or lawfully sufficient for certain effects might in fact not be causes of those effects properly speaking. They also suggest that a failure to appreciate this fact leads many to endorse principles like Causal Closure, when their available evidence only supported weaker principles like Physical Closure. ${ }^{15}$ If, as I will argue, the truth of Causal Closure is not required to generate causal exclusion arguments against non-reductionism, and Physical Closure will do instead, it does not matter, for the purpose of providing exclusion arguments against non-reductionism, that some have confused one for the other.

Physical Exclusion imposes a necessary condition on any non-overdetermining cause occurring at $t$ of an effect, namely not being distinct from any of the effect's physically sufficient phenomena occurring at $t$. The intuition driving Physical Exclusion is that the occurrence of any phenomenon that is distinct from a physically sufficient phenomenon $A$, must be redundant for the occurrence of $B$. Consequently, even if some phenomenon distinct from $A$ were to cause $B$, it would have to do so redundantly. After all, given the occurrence of $A$, the occurrence of $B$ was already settled. So if this distinct phenomenon were to cause $B$, the occurrence of $B$ would be overdetermined by $A$ and the distinct phenomenon.

As opposed to Physical Closure, it is unlikely that many non-reductionists will assent to Physical Exclusion. In order for the overall argument to effectively target standard non-reductionist positions, the 'distinct' in Physical Exclusion should be interpreted as meaning 'non-identical'. Non-reductionists are likely to maintain that the principle is false under this reading, because two phenomena being non-identical still allows for their standing in another relation like supervenience or grounding, and supervening on, or being grounded in, a distinct physically sufficient phenomenon for a

\footnotetext{
15 Menzies (2015) and Zhong (2019) present a similar line of argument.
} 
certain effect should still allow for being a cause of that effect. So the non-reductionist might argue. In the next section, I briefly summarize some of the standard strategies to develop such an objection to Physical Exclusion.

However, we are not concerned here with the ultimate plausibility of Physical Exclusion. What matters for our purpose is that Physical Exclusion avoids the kind of objections that are raised against Causal Exclusion's reliance on causal sufficiency, without creating new problems. If this is indeed the case, and Physical Exclusion can generate a valid exclusion argument against Non-Reductionism, the objections to the notion of causal sufficiency only pose a superficial threat for exclusion arguments. Before turning to a full formulation of an exclusion argument that relies on Physical Exclusion instead of Causal Exclusion, it is worth it setting aside two potential worries about the change from Causal Exclusion to Physical Exclusion.

First, one might worry that this change does not avoid all of the concerns about causal sufficiency. It is often remarked that any effect has a potentially infinite number of sufficient causes at any time preceding the effect. For example, if being male is causally sufficient for not getting pregnant, so is being male and taking birth control, being male and feeling dizzy, etc. ${ }^{16}$ In the context of the exclusion argument, one might worry that there are too many sufficient phenomena to choose from for any effect. Which of these is to exclude distinct phenomena from being causes?

To some extent, these remarks apply to physically sufficient phenomena as well. There appears to be a potentially infinite number of such phenomena for any familiar effect. Start with the maximally specific physical phenomenon spanning the entire cross-section of the backwards light cone of the window shattering and add any redundant phenomenon: physical goings-on outside the backwards light cone, ectoplasmic goings-on inside that light cone, etc. The composite of a physically sufficient phenomenon for the window shattering and an irrelevant phenomenon will also be physically sufficient for the window shattering. ${ }^{17}$

However, Physical Exclusion contains clear instructions as to which physically sufficient phenomena to select: all of them. It states that a cause of an effect cannot be distinct from any of its physically sufficient phenomena. Consequently, physical goings-on outside the backwards light cone and ectoplasmic goings-on inside that light cone are excluded, because they are distinct from at least one physically sufficient phenomenon: the maximally specific physical phenomenon spanning the relevant cross-section of the backwards light cone.

Second, one might worry that the change to Physical Exclusion creates a new problem. In particular, one might object that the resulting overdetermination it posits is not a causal overdetermination. After all, we granted the critics of Causal Exclusion that if $A$ is physically sufficient for $B, A$ is probably not a cause of $B$. Consequently, we cannot conclude that, if $B$ has a cause that is distinct from $A, B$ has one cause too many. Or, to borrow a phrase from Papineau (2002, Section 1.2), we cannot conclude that $B$ is caused twice over. Standard exclusion arguments derive their thrust from the idea that such a systematic causal overdetermination is problematic. Our reformulated

\footnotetext{
16 List and Menzies (2009, p. 489) and Menzies (2013, p. 72) both make this remark and ascribe it to Salmon (1971).

17 After all, $\square(p \rightarrow q)$, entails $\square(p \& r \rightarrow q)$ for any $p, q$ and $r$.
} 
argument does not pose the same threat for non-reductionists, as the resulting overdetermination is not causal. Or so the objection would go. There are two remarks to be made in response to this objection.

First, the resulting overdetermination is objectionable for the same reason that causal overdetermination is taken to be objectionable. The thesis that the effects of mental phenomena are causally overdetermined is taken to be problematic because it would mean that there is a redundant cause for each effect of a mental phenomenon. Such an overdetermination would mean that at least one of its causes does not contribute anything to the occurrence of the effect that was not already contributed by the other cause (cf. Kim 1998, p. 37). Whilst it is possible for causes to be redundant in this way for the occurrence of their effect in special circumstances, such as when two bullets simultaneously hit a victim and each bullet would have killed the victim in the absence of the other, it is wildly implausible that the effects of mental phenomena systematically have causes that are redundant for their occurrence (cf. Papineau 2002, Section 1.5). Given this dialectic, it matters little whether these causes are redundant because of the presence of a distinct cause, or because of the presence of a distinct physically sufficient physical phenomenon. ${ }^{18}$ Either way, it would be a burden on the non-reductionist if her theory states that all mental causes are redundant for the occurrence of their effects.

Second, if $B$ is overdetermined by a physically sufficient physical phenomenon $A$ and a phenomenon that is distinct from $A$, the causal overdetermination of $B$ follows given a plausible further assumption. After all, it is plausible that $A$ contains some phenomenon $a$ that causes $B$ against the background of all the rest of $A$-call that $A-$. By hypothesis, the distinct cause is distinct from both $a$ and $A-$. If that is the case, then $B$ is caused twice over: once by $a$ and once by the distinct cause. After all, both the distinct cause and $a$ cause $B$ against the background of $A-$, and the distinct cause would be redundant: $B$ would have occurred even if the distinct cause were absent. Or so the Physical Exclusion principle would lead us to believe. Consequently, if one endorses Physical Exclusion, and one therefore agrees that the distinct cause and $A$ overdetermine $B$, the causal overdetermination of $B$ follows given an innocent further assumption.

The move from Causal Closure and Causal Exclusion to Physical Closure and Physical Exclusion does not introduce new problems, and it avoids the kinds of objections that are raised against the notion of causal sufficiency at work in Causal Closure and Causal Exclusion. So if Physical Closure and Physical Exclusion can generate a valid exclusion argument, we can conclude that these objections can be answered in a straightforward fashion and therefore do not pose a serious threat to exclusion arguments. In the final section, I provide such a valid exclusion argument, and briefly discuss some worries about its soundness.

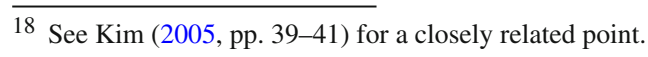




\section{Causal exclusion without causal sufficiency}

The resulting exclusion argument builds on the following set of propositions:

Mental Causation Mental phenomena systematically cause physical phenomena.

Non-Reductionism Mental phenomena are distinct from physical phenomena.

Physical Closure For any physical effect, and any time $t$ there is some physical phenomenon $A$ occurring at $t$ that is physically sufficient for this effect.

Physical Parts For any two phenomena $A$ and $B$, if $A$ is a physical phenomenon and $B$ is a part of $A$, then $B$ is a physical phenomenon as well.

Physical ExclusionFor any three phenomena $A, B$ and $C$ : if $A$ occurs at $t$ and $A$ is physically sufficient for $B$ 's occurrence at $t+x$, no phenomenon $C$ occurring at $t$ that is distinct from $A$ and is distinct from all of $A$ 's parts is a cause of $B$, unless it is a case of genuine overdetermination.

Non-Overdetermination There is no systematic genuine overdetermination of physical effects with mental causes.

This set of propositions is internally inconsistent. Mental Causation and NonReductionism together entail that physical phenomena are systematically caused by phenomena that are distinct from physical phenomena. Physical Closure, Physical Parts and Physical Exclusion together entail that there are no phenomena that are distinct from physical phenomena and cause physical phenomena, unless it is a case of genuine overdetermination. Finally, Non-Overdetermination states that systematic genuine overdetermination is not an option. One of these six has to go. Consequently, the remaining five propositions form a valid exclusion argument against Non-Reductionism.

Of course, it is a further question whether or not the resulting exclusion argument is sound, and there are ongoing debates that affect the plausibility of its premises. Many think that principles like Physical Exclusion are false. Or at least, many think they are false if they exclude non-overdetermining causes that are distinct from physically sufficient phenomena in the same way that most prominent forms of non-reductionists take mental phenomena to be distinct from their underlying physical phenomena. Most non-reductionists hold that mental phenomena are distinct from physical phenomena in the following, limited sense: mental phenomena are not identical to their underlying physical phenomena, but all mental phenomena do metaphysically supervene on their underlying physical phenomena. If Physical Exclusion excludes all phenomena that are distinct from physical phenomena in that sense of being distinct, it meets with some powerful objections. For example, Bennett (2003, 2008) uses a counterfactual test for overdetermination to show that phenomena that metaphysically supervene on, but are non-identical to, physical phenomena can cause effects without overdetermining the occurrence of the effect. Or at least, they would not overdetermine their effects in any problematic sense (cf. Schaffer 2003; Sider 2003). Others have argued that, if a principle like Physical Exclusion is true, then patently causal phenomena like hurricanes, infections and banking crises are excluded from being non-overdetermining causes 
as well; thereby providing a powerful reductio against such exclusion principles (e.g. Block 2003; Bontly 2002). If the exclusionist wants her argument to reject the most prominent forms of non-reductionism, she requires a proper response to these kinds of objections. ${ }^{19}$ Exclusionists have proposed such responses, ${ }^{20}$ and the subsequent debates are ongoing.

However, for our purposes it does not matter which side of these debates is correct. After all, these kinds of objections do not concern the notion of sufficiency at work in the exclusion argument. In fact, authors that formulate such objections often set aside questions concerning the notion of sufficiency. Some do so explicitly, like List and Stoljar (2017, p. 96) and Bennett (2003, p. 490), others do so implicitly, by not addressing the issue at all, like Block (2003), Bontly (2002) and Sider (2003). When criticizing exclusion arguments, these authors do not rely on criticism of the notion of causal sufficiency. If the above reasoning is correct, that is the right attitude. Objections to the role of causal sufficiency in exclusion arguments can be answered by simply reformulating such arguments in terms of physical sufficiency. Therefore, such objections do not pose a serious threat to exclusion arguments. ${ }^{21}$

Acknowledgements Open Access funding provided by Projekt DEAL.

Funding Funding was provided by Deutsche Forschungsgemeinschaft (Grant No. FOR 2495).

Open Access This article is licensed under a Creative Commons Attribution 4.0 International License, which permits use, sharing, adaptation, distribution and reproduction in any medium or format, as long as you give appropriate credit to the original author(s) and the source, provide a link to the Creative Commons licence, and indicate if changes were made. The images or other third party material in this article are included in the article's Creative Commons licence, unless indicated otherwise in a credit line to the material. If material is not included in the article's Creative Commons licence and your intended use is not permitted by statutory regulation or exceeds the permitted use, you will need to obtain permission directly from the copyright holder. To view a copy of this licence, visit http://creativecommons.org/licenses/by/4.0/.

\section{References}

Albert, D. Z. (2015). After physics. Cambridge: Harvard University Press.

Bennett, K. (2003). Why the exclusion problem seems intractable, and how, just maybe, to tract it. Noûs, 37(3), 471-497.

Bennett, K. (2008). Exclusion again. In J. Kallestrup \& J. Hohwy (Eds.), Being reduced (pp. 280-307). Oxford: Oxford University Press.

Bernstein, S. (2016). Overdetermination underdetermined. Erkenntnis, 81(1), 17-40.

Block, N. (2003). Do causal powers drain away? Philosophy and Phenomenological Research, 67(1), $133-150$.

\footnotetext{
19 Some philosophers even extend on such objections to defend full-out dualist theories of the mind, according to which some mental phenomena bear no metaphysically necessary relations to any (collection of) physical phenomena (cf. Chalmers 1996), against exclusion arguments (e.g. Kroedel 2015; List and Stoljar 2017). Bennett (2008) formulates a powerful challenge to such defenses and for now such defenses of dualist mental causation remain non-standard.

20 See, among others, Bernstein (2016), Kim (2005, 2007), Ney (2007, 2012, 2016), Papineau (2013), and Won (2014).

21 I am greatly indebted to Pär Sundström and Torfinn Huvenes for innumerable proofreads and discussions, as well as to Gunnar Björnsson, the audience at the Swedish Congress of Philosophy, and several anonymous referees for thoughtful comments.
} 
Bontly, T. D. (2002). The supervenience argument generalizes. Philosophical Studies, 109(1), 75-96.

Chalmers, D. J. (1996). The conscious mind. Oxford: Oxford University Press.

Crane, T., \& Arnadottir, S. T. (2013). There is no exclusion problem. In S. C. Gibb, E. J. Lowe, \& R. D. Ingthorsson (Eds.), Mental causation and ontology (pp. 248-265). Oxford: Oxford University Press.

Field, H. (2003). Causation in a physical world. In M. J. Loux \& D. W. Zimmerman (Eds.), The Oxford handbook of metaphysics (pp. 435-460). Oxford: Oxford University Press.

Hitchcock, C. (2012). Theories of causation and the causal exclusion argument. Journal of Consciousness Studies, 19(5-6), 40-56.

Johnston, M. (2006). Hylomorphism. Journal of Philosophy, 103(12), 652-698.

Kim, J. (1989). The myth of non-reductive materialism. Proceedings and Addresses of the American Philosophical Association, 63(3), 31-47.

Kim, J. (1998). Mind in a physical world: An essay on the mind-body problem and mental causation. Cambridge: MIT Press.

Kim, J. (2005). Physicalism, or something near enough. Princeton: Princeton University Press.

Kim, J. (2007). Causation and mental causation. In B. P. McLaughlin \& J. D. Cohen (Eds.), Contemporary debates in philosophy of mind (pp. 227-242). Oxford: Blackwell.

Koons, R. C., \& Bealer, G. (2010). Introduction. In R. C. Koons \& G. Bealer (Eds.), The waning of materialism: New essays (pp. 1-31). Oxford: Oxford University Press.

Kroedel, T. (2015). Dualist mental causation and the exclusion problem. Noûs, 49(2), 357-375.

List, C., \& Menzies, P. (2009). Non-reductive physicalism and the limits of the exclusion principle. The Journal of Philosophy, 106(9), 475-502.

List, C., \& Stoljar, D. (2017). Does the exclusion argument put any pressure on dualism? Australasian Journal of Philosophy, 95(1), 96-108.

Loewer, B. M. (2007). Mental causation, or something near enough. In B. P. McLaughlin \& J. D. Cohen (Eds.), Contemporary debates in philosophy of mind (pp. 243-64). Oxford: Blackwell.

Lowe, E. J. (2003). Physical causal closure and the invisibility of mental causation. In S. Walter \& H. Heckmann (Eds.), Physicalism and mental causation (pp. 137-154). Exeter: Imprint Academic.

Menzies, P. (2013). Mental causation in the physical world. In S. Gibb, E. J. Lowe, \& R. Ingthorsson (Eds.), Mental causation and ontology (pp. 58-86). Oxford: Oxford University Press.

Menzies, P. (2015). The causal closure argument is no threat to non-reductive physicalism. Humana Mente, $8(29), 22-46$.

Ney, A. (2007). Can an appeal to constitution solve the exclusion problem? Pacific Philosophical Quarterly, $88(4), 486-506$.

Ney, A. (2012). The causal contribution of mental events. In H. Christopher \& G. Simone (Eds.), New perspectives on type identity: The mental and the physical (pp. 230-250). Cambridge: Cambridge University Press.

Ney, A. (2016). Microphysical causation and the case for physicalism. Analytic Philosophy, 57(1), 141-164.

Papineau, D. (2002). Thinking about consciousness. Oxford: Oxford University Press.

Papineau, D. (2013). Causation is macroscopic but not irreducible. In S. Gibb, E. J. Lowe, \& R. Ingthorsson (Eds.), Mental causation and ontology (pp. 126-151). Oxford: Oxford University Press.

Pernu, T. K. (2013). The principle of causal exclusion does not make sense. Philosophical Forum, 44(1), 89-95.

Pernu, T. K. (2016). Causal exclusion and downward counterfactuals. Erkenntnis, 81(5), 1031-1049.

Raatikainen, P. (2010). Causation, exclusion, and the special sciences. Erkenntnis, 73(3), 349-363.

Raatikainen, P. (2013). Can the mental be causally efficacious? In K. Milkowski \& M. Talmont-Kaminski (Eds.), Regarding the mind, naturally: Naturalist approaches to the sciences of the mental (pp. 138166). Cambridge: Cambridge Scholars Publishing.

Raatikainen, P. (2018). Kim on causation and mental causation. E-Logos Electronic Journal for Philosophy, 25(2), 22-47.

Salmon, W. (1971). Statistical explanation \& statistical relevance. Pittsburgh: University of Pittsburgh Press.

Schaffer, J. (2003). Overdetermining causes. Philosophical Studies, 114(1-2), 23-45.

Shapiro, L. (2010). Lessons from causal exclusion. Philosophy and Phenomenological Research, 81(3), 594-604.

Shapiro, L. (2012). Mental manipulations and the problem of causal exclusion. Australasian Journal of Philosophy, 90(3), 507-524. 
Shapiro, L., \& Sober, E. (2007). Epiphenomenalism. The do's and don'ts. In G. Wolters \& P. Machamer (Eds.), Thinking about causes: From Greek philosophy to modern physics (pp. 235-264). Pittsburgh: University of Pittsburgh Press.

Sider, T. (2003). Review: What's so bad about overdetermination? Philosophy and Phenomenological Research, 67(3), 719-726.

Won, C. (2014). Overdetermination, counterfactuals, and mental causation. Philosophical Review, 123(2), 205-229.

Won, C. (2019). Mental causation as joint causation. Synthese. https://doi.org/10.1007/s11229-01902378-4.

Woodward, J. (2008). Mental causation and neural mechanisms. In J. Kallestrup \& J. Hohwy (Eds.), Being reduced (pp. 218-263). Oxford: Oxford University Press.

Zhong, L. (2019). Taking emergentism seriously. Australasian Journal of Philosophy, 98(1), 31-46.

Publisher's Note Springer Nature remains neutral with regard to jurisdictional claims in published maps and institutional affiliations. 